young children in Scotland, suggesting disadvantaged SEC are associated with higher prescription use. Inequalities in ADHD prescriptions across childhood may not be fully captured since prevalence increases with age (and our data only follow children up to the maximum age of 8). Prescription data may underestimate prevalence of $\mathrm{ADHD}$ as not all children with ADHD symptoms will be diagnosed and/or prescribed (and this may vary by SEC). Future analyses will explore this using data from child health checks.

\section{RF29 VIOLENCE AGAINST PEOPLE WITH DISABILITES: VARIATIONS BY IMPAIRMENT TYPE, GENDER AND SOCIO-ECONOMIC STATUS IN AN AUSTRALIAN POPULATION-BASED STUDY}

'L Krnjacki*, ${ }^{2} E$ Emerson, ${ }^{2} \mathrm{G}$ Llewellyn, 'Z Aitken, 'A Kavanagh. ' Centre for Health Equity, The University of Melbourne, Melbourne, Australia; ${ }^{2}$ Centre for Disability Research and Policy, The University of Sydney, Sydney, Australia

\subsection{6/jech-2019-SSMabstracts. 144}

Background Recent international meta-analyses show that children and adults with disabilities are more likely to experience interpersonal violence than those without disabilities. People with disabilities are a heterogeneous group, and further work is required to determine the variations in the experience of violence by impairment type. It is also important to assess the extent to which any between-group differences may be explained by socio-economic situation.

Methods We analysed the 2015 Australian Bureau of Statistics Survey on Personal Safety of more than 21,000 adults. We used population-weighted, age-adjusted, logistic regression to estimate the odds of violence in the last 12 months by impairment type and gender. We stratified by five forms of violence (physical, sexual and intimate partner, emotional abuse and stalking/harassment) and for specific impairment types (physical, intellectual, psychological and sensory). We also investigated potential effect modification by poverty status.

Results Results for women showed that those with sensory and physical impairments fared the best, with a two-fold increase in odds across all five forms of violence. Women with intellectual and psychological impairments fared worse for all forms of violence. There was a three-fold increase in the odds for emotional abuse and stalking/harassment. There was an even greater increase in the odds for sexual, physical and partner violence. Women with intellectual impairments had between a five and seven-fold increase in the odds of these forms of violence, while women with psychological impairments had been a four and six-fold increase. All results were statistically significant at $\mathrm{p}=0.05$.

Results for men showed much less variation by impairment type. There was a statistically significant two-fold increase in odds of physical violence for those with sensory, intellectual and psychological impairments. There were no significant differences between men with impairments compared to men without disabilities for sexual violence, partner violence and emotional abuse. There was evidence that stalking/harassment was worse for men with psychological impairments.
Some of these associations were moderated by poverty status, with the increased odds of exposure to violence among people with disabilities being greater for people living in poverty.

Conclusion These results show there are important variations in the experience of violence for people with disability depending on their impairment type, gender and socio-economic status. There is a clear need to develop interventions that are targeted to the particular circumstances and needs of these high-risk groups.

\section{RF30 RESIDENTIAL AREA DEPRIVATION PREDICTS SUBSEQUENT HOSPITAL ADMISSION IN A BRITISH POPULATION INDEPENDENTLY OF SOCIAL CLASS AND EDUCATION STATUS: THE EPIC-NORFOLK COHORT}

${ }^{1}$ RN Luben*, 'SA Hayat, ${ }^{2} \mathrm{NJ}$ Wareham, ${ }^{1} \mathrm{PD}$ Pharoah, ${ }^{1} \mathrm{KT}$ Khaw. ${ }^{1}$ Public Health and Primary Care, University of Cambridge, Cambridge, UK; ${ }^{2}$ MRC Epidemiology Unit, University of Cambridge, Cambridge, UK

\subsection{6/jech-2019-SSMabstracts. 145}

Background Low social economic position is linked to higher rates of mortality and morbidity. Socioeconomic factors are reported to predict admission to hospital for many conditions. It is less clear if residential area deprivation index predicts hospital usage independently of individual social class and lifestyle factors.

Methods We examined the relationship between residential Townsend's Area Deprivation Index and subsequent admissions to hospital and time spent in hospital for 11,214 men and 13,763 women aged 40-79 years in the general population.

Participants from the EPIC-Norfolk prospective populationbased study were followed for nineteen years (1999-2018) using record linkage. Townsend's Index from the small area measurements taken at the 1991 UK census was linked to individuals in the EPIC-Norfolk cohort using their postal code.

Results Compared to those with residential Townsend Area Deprivation Index lower than the average for England and Wales, those with a higher than average deprivation index had a higher likelihood of spending more than twenty days in hospital multivariable adjusted odds ratio (OR) 1.18 (95\% confidence interval (CI) 1.07-1.29) and having 7 or more admissions OR 1.11 (95\% CI 1.02-1.22) after adjustment for age, sex, smoking status, education, social class, body mass index and prevalent diseases. Those with manual social class were at greater risk of hospitalisation compared to those with a non-manual social class when living in an area with higher deprivation index $(p$ Interaction $=0.025)$. Similarly, those with a lower education level living in a more deprived area had a higher risk of hospitalisation ( $\mathrm{p}$ Interaction $=0.020$ ) when compared with those with a higher education level.

Conclusion Residential area deprivation predicts future hospitalisations - time spent in hospital and number of admissions independently of individual social class and education level and other behavioural factors. There are significant interactions such that residential area deprivation has greater impact in those with low education level or manual social class. 